\title{
One-loop QCD corrections to gluon-gluon scattering at NNLO*
}

\author{
E. W. N. Glover ${ }^{a}$ and M. E. Tejeda-Yeomans ${ }^{a}$ \\ ${ }^{a}$ Department of Physics, University of Durham, Durham DH1 3LE, England \\ E-mail: E.W.N.Glover@durham.ac.uk, M.E.Tejeda-Yeomans@durham.ac.uk
}

\begin{abstract}
We present the $\mathcal{O}\left(\alpha_{s}^{4}\right)$ virtual QCD corrections to gluon-gluon scattering due to the self-interference of the one-loop amplitude. We give analytic expressions renormalised in the $\overline{\mathrm{MS}}$ scheme and work in conventional dimensional regularisation. We write the structure of the infrared divergences from direct Feynman diagram evaluation in terms of the Catani formalism for infrared divergences. Formulae for the finite remainder are given in terms of logarithms that are real in the physical region. These results, together with those previously obtained for quark-quark and quark-gluon scattering complete the one-loop matrix elements for $2 \rightarrow 2$ processes needed for the next-to-next-to-leading order contribution to inclusive jet production at hadron colliders.
\end{abstract}

KeYwords: QCD, Jets, LEP HERA and SLC Physics, NLO and NNLO Computations.

\footnotetext{
${ }^{*}$ Work supported in part by the UK Particle Physics and Astronomy Research Council and by the EU Fourth Framework Programme 'Training and Mobility of Researchers', Network 'Quantum Chromodynamics and the Deep Structure of Elementary Particles', contract FMRX-CT98-0194 (DG 12 - MIHT). M.E.T. acknowledges financial support from CONACyT and the CVCP. We thank the British Council and German Academic Exchange Service for support under ARC project 1050 .
} 


\section{Introduction}

To improve our understanding of jet production in current and future high energy collider experiments we need more accurate perturbative calculations in quantum chromodynamics (QCD). The progress of recent years means that nowadays a Nextto-Leading Order (NLO) perturbative calculation is standard (see for example [1, 2]). Developments of efficient techniques for the evaluation of matrix elements and numerical algorithms that allow the virtual and bremstrahlung contributions to be combined have made NLO perturbative calculations commonplace. However, new improvements in detector technology and an increase on statistics and luminosity require more precise theoretical calculations. The reduction of theoretical uncertainties is crucial to refine our understanding of QCD and to identify signatures of possible new physics beyond the Standard Model.

The calculation of fixed next-to-next-to-leading order (NNLO) virtual corrections for massless $2 \rightarrow 2$ processes is now an attainable task since a vital breakthough has been made in the calculation of two-loop master integrals $[3,4,5,6,7,8,9$, $10,11]$, in methods for reducing tensor integrals to master integrals $[5,6,9]$ and in the prediction of the structure of infrared divergences [12]. The first two-loop contributions for light-like $2 \rightarrow 2$ scattering were addressed by Bern, Dixon together with Kosower [13] and Ghinculov [14] who have calculated the maximal-helicityviolating two-loop amplitude for $g g \rightarrow g g$, and the QED processes $e^{+} e^{-} \rightarrow \mu^{+} \mu^{-}$ and $e^{+} e^{-} \rightarrow e^{-} e^{+}$, respectively. More recently, Gehrmann and Remiddi [15] have provided analytic expressions for the complete set of two-loop integrals with one offshell leg. These integrals are sufficient to allow the two-loop amplitudes for three jet production in $e^{+} e^{-}$annihilation to be evaluated.

Recently, we provided analytical expressions for the $\mathcal{O}\left(\alpha_{s}^{4}\right)$ one-loop and twoloop contribution to quark-quark scattering $[16,17,18]$, and quark-gluon scattering [19], as well as crossed and time-reversed processes, in the limit where the quark mass is neglected. The $\mathcal{O}\left(\alpha_{s}^{4}\right)$ two-loop contribution to gluon scattering is given in Ref. [20]. To complete the list of ingredients needed for the NNLO virtual corrections to gluon-gluon scattering, the interference term of one-loop amplitude with one-loop amplitude needs to be included. In this paper we present analytical formulae for this contribution using conventional dimensional regularisation (CDR) and renormalised in the $\overline{\mathrm{MS}}$ scheme.

The organisation of our paper is the following. We provide our notation and describe our method briefly in Sec. 2. In Section 3 we give analytic expressions for the interference of one-loop amplitude with one-loop amplitude. In Sec. 3.1 we follow closely the notation used in Refs. $[12,20]$ to write the infrared singularity structure in the $\overline{\mathrm{MS}}$ scheme for the one-loop contribution to the NNLO calculation in terms of the one-loop bubble and the one-loop box integral given in Appendix A. We show that the anticipated singularity structure agrees with our explicit calculation. 
Finally, in Sec. 3.2 we give the finite contribution in terms of logarithms that have no imaginary parts. We conclude with a brief summary of results.

\section{Notation}

As in Ref. [20] we consider the scattering process

$$
g\left(p_{1}\right)+g\left(p_{2}\right)+g\left(p_{3}\right)+g\left(p_{4}\right) \rightarrow 0
$$

where the gluons are all incoming with light-like momenta and carry colour indexes, $a_{i}$, in the adjoint representation.

Their total momentum is conserved and satisfies

$$
p_{1}^{\mu}+p_{2}^{\mu}+p_{3}^{\mu}+p_{4}^{\mu}=0, \quad p_{i}^{2}=0,
$$

where the associated Mandelstam variables are given by

$$
s=\left(p_{1}+p_{2}\right)^{2}, \quad t=\left(p_{2}+p_{3}\right)^{2}, \quad u=\left(p_{1}+p_{3}\right)^{2}, \quad s+t+u=0 .
$$

We treat all external gluon states in $D$ dimensions, i.e. we use CDR, and we renormalise the ultraviolet divergences in the $\overline{\mathrm{MS}}$ scheme. In this scheme, the renormalised four point amplitude can be written as

$$
|\mathcal{M}\rangle=4 \pi \alpha_{s}\left[\left|\mathcal{M}^{(0)}\right\rangle+\left(\frac{\alpha_{s}}{2 \pi}\right)\left|\mathcal{M}^{(1)}\right\rangle+\left(\frac{\alpha_{s}}{2 \pi}\right)^{2}\left|\mathcal{M}^{(2)}\right\rangle+\mathcal{O}\left(\alpha_{s}^{3}\right)\right]
$$

where $\alpha_{s} \equiv \alpha_{s}\left(\mu^{2}\right)$ is the running coupling at renormalisation scale $\mu$ and the $\left|\mathcal{M}^{(i)}\right\rangle$ represents the colour-space vector describing the renormalised $i$-loop amplitude. The dependence on both renormalisation scale $\mu$ and renormalisation scheme is implicit.

The squared amplitude summed over spins and colours is denoted as

$$
\mathcal{D}(s, t, u)=\langle\mathcal{M} \mid \mathcal{M}\rangle=\sum|\mathcal{M}(g+g \rightarrow g+g)|^{2},
$$

which is symmetric under the exchange of $s, t$ and $u$.

Following from Eq. (2.3), the function $\mathcal{D}$ can be expanded perturbatively to yield

$$
\mathcal{D}(s, t, u)=16 \pi^{2} \alpha_{s}^{2}\left[\mathcal{D}^{4}(s, t, u)+\left(\frac{\alpha_{s}}{2 \pi}\right) \mathcal{D}^{6}(s, t, u)+\left(\frac{\alpha_{s}}{2 \pi}\right)^{2} \mathcal{D}^{8}(s, t, u)+\mathcal{O}\left(\alpha_{s}^{3}\right)\right]
$$

where

$$
\begin{aligned}
\mathcal{D}^{4}(s, t, u) & =\left\langle\mathcal{M}^{(0)} \mid \mathcal{M}^{(0)}\right\rangle \\
& =16 V N^{2}(1-\epsilon)^{2}\left(3-\frac{u t}{s^{2}}-\frac{u s}{t^{2}}-\frac{s t}{u^{2}}\right) \\
\mathcal{D}^{6}(s, t, u) & =\left\langle\mathcal{M}^{(0)} \mid \mathcal{M}^{(1)}\right\rangle+\left\langle\mathcal{M}^{(1)} \mid \mathcal{M}^{(0)}\right\rangle \\
\mathcal{D}^{8}(s, t, u) & =\left\langle\mathcal{M}^{(1)} \mid \mathcal{M}^{(1)}\right\rangle+\left\langle\mathcal{M}^{(0)} \mid \mathcal{M}^{(2)}\right\rangle+\left\langle\mathcal{M}^{(2)} \mid \mathcal{M}^{(0)}\right\rangle
\end{aligned}
$$


and $V=N^{2}-1$ with $N$ the number of colours. Expressions for $\mathcal{D}^{6}$ are given in Ref. [21] using CDR to isolate the infrared and ultraviolet singularities. Also, analytic formulae for the two-loop contribution to $\mathcal{D}^{8}$

$$
\mathcal{D}^{8(2 \times 0)}(s, t, u)=\left\langle\mathcal{M}^{(0)} \mid \mathcal{M}^{(2)}\right\rangle+\left\langle\mathcal{M}^{(2)} \mid \mathcal{M}^{(0)}\right\rangle
$$

are given in Ref. [20].

We now present expressions for the infrared singular and finite contributions to $\mathcal{D}^{8}$ due to the interference of one-loop amplitudes with one-loop amplitudes, namely

$$
\mathcal{D}^{8(1 \times 1)}(s, t, u)=\left\langle\mathcal{M}^{(1)} \mid \mathcal{M}^{(1)}\right\rangle \text {. }
$$

This contribution is somewhat simpler to evaluate than the two-loop contribution but it is a vital part of the NNLO virtual corrections and we present it here for completeness.

As in Refs. [16, 17, 18, 19, 20], we use QGRAF [22] to produce the one-loop Feynman diagrams to construct $\left|\mathcal{M}^{(1)}\right\rangle$. We perform the internal trace (for fermion loops) over Dirac matrices in $D$ dimensions. The tensor integrals that emerge from this amplitude are associated with scalar integrals in higher dimensions and with higher powers of propagators $[9,23]$.

These integrals, in turn, can be reduced to master integrals in $D=4-2 \epsilon$ with the systematic application of integration-by-parts identities [24]. At the end, all integrals arising in the one-loop amplitudes can be written in terms of the one-loop bubble integral in $D=4-2 \epsilon$ and the finite one-loop box integral in $D=6-2 \epsilon$ (see Appendix for expansions around $\epsilon=0$ ). This choice of master integrals is not unique but is useful to have a natural separation of the infrared poles and the finite part of the one-loop amplitudes.

Finally, we project by $\left\langle\mathcal{M}^{(1)}\right|$ and perform the summation over colours and spins. Note that when we sum over the gluon polarisations, we ensure that the polarisation states are transversal by the use of an axial gauge

$$
\sum_{\text {spins }} \epsilon_{i}^{\mu} \epsilon_{i}^{\nu *}=-g^{\mu \nu}+\frac{n_{i}^{\mu} p_{i}^{\nu}+n_{i}^{\nu} p_{i}^{\mu}}{n_{i} \cdot p_{i}}
$$

where $p_{i}$ is the momentum, $\epsilon_{i}$ is the polarisation vector and $n_{i}$ is an arbitrary lightlike 4 -vector for gluon $i$. For simplicity, we choose $n_{1}^{\mu}=p_{2}^{\mu}, n_{2}^{\mu}=p_{1}^{\mu}, n_{3}^{\mu}=p_{4}^{\mu}$ and $n_{4}^{\mu}=p_{3}^{\mu}$.

\section{One-loop contribution}

We further decompose the one-loop contributions as a sum of two terms

$$
\mathcal{D}^{8(1 \times 1)}(s, t, u)=\mathcal{P} \text { oles }(s, t, u)+\mathcal{F} \text { inite }(s, t, u) .
$$


Poles contains infrared singularities that will be analytically canceled by those occurring in radiative processes of the same order (ultraviolet divergences are removed by renormalisation). Finite is the remainder which is finite as $\epsilon \rightarrow 0$.

\subsection{Infrared Pole Structure}

Following the procedure outlined in Refs. [12, 20], we can write the infrared pole structure of the one-loop contributions renormalised in the $\overline{\mathrm{MS}}$ scheme as

$$
\begin{aligned}
\text { Poles }= & 2 \operatorname{Re}\left[-\frac{1}{2}\left\langle\mathcal{M}^{(0)}\left|\boldsymbol{I}^{(1) \dagger}(\epsilon) \boldsymbol{I}^{(1)}(\epsilon)\right| \mathcal{M}^{(0)}\right\rangle\right. \\
& \left.-\frac{\beta_{0}}{\epsilon}\left\langle\mathcal{M}^{(0)}\left|\boldsymbol{I}^{(1)}(\epsilon)\right| \mathcal{M}^{(0)}\right\rangle+\left\langle\mathcal{M}^{(1, u n)}\left|\boldsymbol{I}^{(1)}(\epsilon)\right| \mathcal{M}^{(0)}\right\rangle\right]
\end{aligned}
$$

where $\left|\mathcal{M}^{(0)}\right\rangle$ is the tree amplitude and $\left|\mathcal{M}^{(1, u n)}\right\rangle$ is the unrenormalised one-loop amplitude, obtained by direct Feynman diagram evaluation. Also, the first coefficient of the QCD beta function for $N_{F}$ (massless) quark flavours is

$$
\beta_{0}=\frac{11 C_{A}-4 T_{R} N_{F}}{6} \quad \text { with } \quad C_{A}=N, \quad T_{R}=\frac{1}{2} .
$$

It is convenient to decompose $\left|\mathcal{M}^{(0)}\right\rangle$ and $\left|\mathcal{M}^{(1, u n)}\right\rangle$ in terms of $S U(N)$ matrices in the fundamental representation, $T^{a},[25,26,27,28,29]$ so that $\left|\mathcal{M}^{(0)}\right\rangle$ and $\left|\mathcal{M}^{(1, u n)}\right\rangle$ may be expressed as nine-dimensional vectors in colour space

$$
\begin{aligned}
\left|\mathcal{M}^{(0)}\right\rangle & =\left(\mathcal{T}_{1}, \mathcal{T}_{2}, \mathcal{T}_{3}, \mathcal{T}_{4}, \mathcal{T}_{5}, \mathcal{T}_{6}, 0,0,0\right)^{T} \\
\left|\mathcal{M}^{(1, u n)}\right\rangle & =\left(\mathcal{L}_{1}, \mathcal{L}_{2}, \mathcal{L}_{3}, \mathcal{L}_{4}, \mathcal{L}_{5}, \mathcal{L}_{6}, \mathcal{L}_{7}, \mathcal{L}_{8}, \mathcal{L}_{9}\right)^{T}
\end{aligned}
$$

where ${ }^{T}$ indicates the transpose vector. Here the tree and loop amplitudes $\mathcal{T}_{i}$ and $\mathcal{L}_{i}$ are the components of $\left|\mathcal{M}^{(0)}\right\rangle$ and $\left|\mathcal{M}^{(1, u n)}\right\rangle$ in the colour space spanned by the (non-orthogonal) basis

$$
\begin{aligned}
& \mathcal{C}_{1}=\operatorname{Tr}\left(T^{a_{1}} T^{a_{2}} T^{a_{3}} T^{a_{4}}\right), \\
& \mathcal{C}_{2}=\operatorname{Tr}\left(T^{a_{1}} T^{a_{2}} T^{a_{4}} T^{a_{3}}\right), \\
& \mathcal{C}_{3}=\operatorname{Tr}\left(T^{a_{1}} T^{a_{4}} T^{a_{2}} T^{a_{3}}\right), \\
& \mathcal{C}_{4}=\operatorname{Tr}\left(T^{a_{1}} T^{a_{3}} T^{a_{2}} T^{a_{4}}\right), \\
& \mathcal{C}_{5}=\operatorname{Tr}\left(T^{a_{1}} T^{a_{3}} T^{a_{4}} T^{a_{2}}\right), \\
& \mathcal{C}_{6}=\operatorname{Tr}\left(T^{a_{1}} T^{a_{4}} T^{a_{3}} T^{a_{2}}\right), \\
& \mathcal{C}_{7}=\operatorname{Tr}\left(T^{a_{1}} T^{a_{2}}\right) \operatorname{Tr}\left(T^{a_{3}} T^{a_{4}}\right), \\
& \mathcal{C}_{8}=\operatorname{Tr}\left(T^{a_{1}} T^{a_{3}}\right) \operatorname{Tr}\left(T^{a_{2}} T^{a_{4}}\right), \\
& \mathcal{C}_{9}=\operatorname{Tr}\left(T^{a_{1}} T^{a_{4}}\right) \operatorname{Tr}\left(T^{a_{2}} T^{a_{3}}\right) .
\end{aligned}
$$


Note that the amplitudes themselves are not required since we compute the interference of tree and loop amplitudes directly.

In the same colour basis, the infrared-singularity operator $\boldsymbol{I}^{(1)}(\epsilon)$ introduced by Catani [12] has the form [20]

$$
\begin{aligned}
& \boldsymbol{I}^{(1)}(\epsilon)=-\frac{e^{\epsilon \gamma}}{\Gamma(1-\epsilon)}\left(\frac{1}{\epsilon^{2}}+\frac{\beta_{0}}{N \epsilon}\right) \\
& \times\left(\begin{array}{ccccccccc}
N(\mathrm{~S}+\mathrm{T}) & 0 & 0 & 0 & 0 & 0 & (\mathrm{~T}-\mathrm{U}) & 0 & (\mathrm{~S}-\mathrm{U}) \\
0 & N(\mathrm{~S}+\mathrm{U}) & 0 & 0 & 0 & 0 & (\mathrm{U}-\mathrm{T}) & (\mathrm{S}-\mathrm{T}) & 0 \\
0 & 0 & N(\mathrm{~T}+\mathrm{U}) & 0 & 0 & 0 & 0 & (\mathrm{~T}-\mathrm{S}) & (\mathrm{U}-\mathrm{S}) \\
0 & 0 & 0 & N(\mathrm{~T}+\mathrm{U}) & 0 & 0 & 0 & (\mathrm{~T}-\mathrm{S}) & (\mathrm{U}-\mathrm{S}) \\
0 & 0 & 0 & 0 & N(\mathrm{~S}+\mathrm{U}) & 0 & (\mathrm{U}-\mathrm{T}) & (\mathrm{S}-\mathrm{T}) & 0 \\
0 & 0 & 0 & 0 & 0 & N(\mathrm{~S}+\mathrm{T}) & (\mathrm{T}-\mathrm{U}) & 0 & (\mathrm{~S}-\mathrm{U}) \\
(\mathrm{S}-\mathrm{U}) & (\mathrm{S}-\mathrm{T}) & 0 & 0 & (\mathrm{~S}-\mathrm{T}) & (\mathrm{S}-\mathrm{U}) & 2 N \mathrm{~S} & 0 & 0 \\
0 & (\mathrm{U}-\mathrm{T}) & (\mathrm{U}-\mathrm{S}) & (\mathrm{U}-\mathrm{S}) & (\mathrm{U}-\mathrm{T}) & 0 & 0 & 2 N \mathrm{U} & 0 \\
(\mathrm{~T}-\mathrm{U}) & 0 & (\mathrm{~T}-\mathrm{S}) & (\mathrm{T}-\mathrm{S}) & 0 & (\mathrm{~T}-\mathrm{U}) & 0 & 0 & 2 N \mathrm{~T}
\end{array}\right)
\end{aligned}
$$

where

$$
\mathrm{S}=\left(-\frac{\mu^{2}}{s+i 0}\right)^{\epsilon}, \quad \mathrm{T}=\left(-\frac{\mu^{2}}{t}\right)^{\epsilon}, \quad \mathrm{U}=\left(-\frac{\mu^{2}}{u}\right)^{\epsilon} .
$$

The matrix $\boldsymbol{I}^{(1)}(\epsilon)$ acts directly as a rotation matrix on $\left|\mathcal{M}^{(0)}\right\rangle$ and $\left|\mathcal{M}^{(1, u n)}\right\rangle$ in colour space. Note that on expanding $\mathrm{S}$, imaginary parts are generated, the sign of which is fixed by the small imaginary part $+i 0$. Other combinations such as $\left\langle\mathcal{M}^{(0)}\right| \boldsymbol{I}^{(1) \dagger}(\epsilon)$ are obtained using the hermitian conjugate operator $\boldsymbol{I}^{(1) \dagger}(\epsilon)$ where the only practical change is that the sign of the imaginary part of $\mathrm{S}$ is reversed.

The contraction of the colour vector $|X\rangle$ with the conjugate colour vector $\langle Y|$ obeys the rule

$$
\langle Y \mid X\rangle=\sum_{\text {spins }} \sum_{\text {colours }} \sum_{i, j=1}^{9} Y_{i}^{*} X_{j} \mathcal{C}_{i}^{*} \mathcal{C}_{j}
$$

In evaluating these contractions, we typically encounter $\sum_{\text {colours }} \mathcal{C}_{i}^{*} \mathcal{C}_{j}$ which is given by the $i j$ component of the symmetric matrix $\mathcal{C C}$

$$
\mathcal{C}=\frac{V}{16 N^{2}}\left(\begin{array}{ccccccccc}
C_{1} & C_{2} & C_{2} & C_{2} & C_{2} & C_{3} & N V & -N & N V \\
C_{2} & C_{1} & C_{2} & C_{2} & C_{3} & C_{2} & N V & N V & -N \\
C_{2} & C_{2} & C_{1} & C_{3} & C_{2} & C_{2} & -N & N V & N V \\
C_{2} & C_{2} & C_{3} & C_{1} & C_{2} & C_{2} & -N & N V & N V \\
C_{2} & C_{3} & C_{2} & C_{2} & C_{1} & C_{2} & N V & N V & -N \\
C_{3} & C_{2} & C_{2} & C_{2} & C_{2} & C_{1} & N V & -N & N V \\
N V & N V & -N & -N & N V & N V & N^{2} V & N^{2} & N^{2} \\
-N & N V & N V & N V & N V & -N & N^{2} & N^{2} V & N^{2} \\
N V & -N & N V & N V & -N & N V & N^{2} & N^{2} & N^{2} V
\end{array}\right),
$$


with

$$
C_{1}=N^{4}-3 N^{2}+3, \quad C_{2}=3-N^{2}, \quad C_{3}=3+N^{2} .
$$

Similarly, we find that the interference of the tree-level amplitudes $\sum_{\text {spins }} \mathcal{T}_{i}^{*} \mathcal{T}_{j}$ is given by $\mathcal{T T}_{i j}$, where

$$
\mathcal{T} \mathcal{T}=\frac{64(1-\epsilon)^{2}\left(t^{2}+u t+u^{2}\right)^{2}}{s^{2} t^{2} u^{2}} \mathcal{V}^{T} \mathcal{V}
$$

and the vector $\mathcal{V}$ is

$$
\mathcal{V}=(u, t, s, s, t, u, 0,0,0)
$$

Also, the interference of the tree-level amplitudes with one-loop amplitudes $\sum_{\text {spins }} \mathcal{T}_{i}^{*} \mathcal{L}_{j}$ is given by $\mathcal{T L}_{i j}$, where

$$
\mathcal{T L}=\mathcal{V}^{T} \mathcal{W}
$$

and the vector $\mathcal{W}$ is

$$
\mathcal{W}=(\mathcal{F}(s, t), \mathcal{F}(s, u), \mathcal{F}(u, t), \mathcal{F}(u, t), \mathcal{F}(s, u), \mathcal{F}(s, t), \mathcal{G}, \mathcal{G}, \mathcal{G})
$$

The function $\mathcal{F}(s, t)$ is symmetric under the exchange of $s$ and $t$, while $\mathcal{G}$ is symmetric under the exchange of any two Mandelstam invariants, so that

$$
\begin{aligned}
\mathcal{F}(s, t) & =f_{1}(s, t, u)+f_{1}(t, s, u), \\
\mathcal{G} & =f_{2}(s, t, u)+f_{2}(s, u, t)+f_{2}(t, s, u)+f_{2}(t, u, s)+f_{2}(u, s, t)+f_{2}(u, t, s) .
\end{aligned}
$$

Here $f_{1}$ and $f_{2}$ are given in terms of the one-loop box integral in $D=6-2 \epsilon$ dimensions and the one-loop bubble graph in $D=4-2 \epsilon$ (see Appendix A),

$$
\begin{aligned}
f_{1}(s, t, u)= & \frac{16 N(1-2 \epsilon)}{s^{2} t^{2}}\left[2(1-\epsilon)^{2}\left(s^{4}+s^{3} t+s t^{3}+t^{4}\right)+3(1-5 \epsilon) s^{2} t^{2}\right] \operatorname{Box}^{6}(s, t) \\
+ & \frac{8 N_{F}(1-2 \epsilon)}{s t}\left[(1-\epsilon)^{2}\left(s^{2}+t^{2}\right)+\epsilon(1+3 \epsilon) s t\right] \operatorname{Box}^{6}(s, t) \\
- & \frac{16 N(1-\epsilon)}{s^{2} t^{2} u \epsilon(3-2 \epsilon)}\left[\left(12-22 \epsilon+12 \epsilon^{2}+2 \epsilon^{3}\right) s^{4}+\left(24-58 \epsilon+50 \epsilon^{2}-6 \epsilon^{3}-2 \epsilon^{4}\right) s^{3} t\right. \\
& \quad+\left(36-99 \epsilon+93 \epsilon^{2}-24 \epsilon^{3}-2 \epsilon^{4}\right) s^{2} t^{2}+(1-\epsilon)\left(24-50 \epsilon+23 \epsilon^{2}\right) s t^{3} \\
& \left.+4(1-\epsilon)(1-2 \epsilon)(3-2 \epsilon) t^{4}\right] \operatorname{Bub}(t) \\
+ & \frac{16 N_{F}}{s t^{2} u(3-2 \epsilon)}\left[\left(4-12 \epsilon+16 \epsilon^{2}-4 \epsilon^{3}\right) s^{3}+\left(3-10 \epsilon+23 \epsilon^{2}-8 \epsilon^{3}\right) s^{2} t\right. \\
\left.\quad+\left(6-15 \epsilon+21 \epsilon^{2}-8 \epsilon^{3}\right) s t^{2}+(1-\epsilon)\left(5-6 \epsilon+2 \epsilon^{2}\right) t^{3}\right] \operatorname{Bub}(t), & \\
f_{2}(s, t, u)= & \frac{32(1-2 \epsilon)}{u^{2}}\left[-4(1-\epsilon)^{2} s t+3(1-5 \epsilon) u^{2}\right] \operatorname{Box}(u, t) \\
& +\frac{32(1-\epsilon)}{\epsilon s u^{2}}\left[4(1-2 \epsilon)(1-\epsilon) t^{2}+(8-17 \epsilon)(1-\epsilon) u t\right. \\
\quad & \left.\left(6-20 \epsilon+15 \epsilon^{2}+\epsilon^{3}\right) u^{2}\right] \operatorname{Bub}(s) .
\end{aligned}
$$


It can be easily noted that the leading infrared singularity in Eq. (3.2) is $\mathcal{O}\left(1 / \epsilon^{4}\right)$. It is a very stringent check on the reliability of our calculation that the pole structure obtained by computing the Feynman diagrams directly and introducing series expansions in $\epsilon$ for the scalar master integrals agrees with Eq. (3.2) through to $\mathcal{O}(1 / \epsilon)$. We therefore construct the finite remainder by subtracting Eq. (3.2) from the full result.

\subsection{Finite contributions}

The finite two-loop contribution to $\mathcal{D}^{8}(s, t, u)$ is defined as

$$
\text { Finite }(s, t, u)=\mathcal{D}^{8(1 \times 1)}(s, t, u)-\mathcal{P} \text { oles }(s, t, u),
$$

where we subtract the series expansions of both $\mathcal{D}^{8(1 \times 1)}(s, t, u)$ and $\mathcal{P}$ oles $(s, t, u)$ and set $\epsilon \rightarrow 0$.

For convenience, we introduce the following logarithms

$$
X=\log \left(\frac{-t}{s}\right), \quad Y=\log \left(\frac{-u}{s}\right), \quad S=\log \left(\frac{s}{\mu^{2}}\right),
$$

where $\mu$ is the renormalisation scale.

We choose to present our results by grouping terms according to the power of the number of colours $N$ and the number of light quarks $N_{F}$, so that

$$
\text { Finite }(s, t, u)=V\left(N^{4} A+N^{2} B+N^{3} N_{F} C+N N_{F} D+N^{2} N_{F}^{2} E+N_{F}^{2} F+\frac{N_{F}^{2}}{N^{2}} G\right) \text {, }
$$

where

$$
\begin{aligned}
A=\{ & \frac{1}{2}\left(X^{2}-2 X Y+Y^{2}+\pi^{2}\right)\left(X^{2}-2 X Y-2 X+Y^{2}+2 Y+\pi^{2}\right) \frac{t^{4}}{s^{4}} \\
& +\left(3 X^{4}-4 X^{3} Y-\frac{56}{3} X^{3}+6 X^{2} Y^{2}+20 X^{2} Y-\frac{22}{3} X^{2} S+10 X^{2} \pi^{2}+\frac{56}{9} X^{2}\right. \\
& -4 X Y^{3}-20 X Y^{2}-4 X Y \pi^{2}-6 X Y+\frac{154}{9} X S-16 X \pi^{2}+\frac{785}{27} X+Y^{4} \\
& +4 Y^{3}-\frac{22}{3} Y^{2} S+2 Y^{2} \pi^{2}-\frac{28}{9} Y^{2}+\frac{110}{3} Y S+16 Y \pi^{2}+\frac{721}{9} Y+\frac{242}{9} S^{2} \\
& \left.+\frac{2948}{27} S+\pi^{4}+\pi^{2}+\frac{9014}{81}\right) \frac{t^{2}}{s^{2}} \\
& +\left(4 X^{4}+12 X^{3}+\frac{4}{3} X^{2} Y-\frac{44}{3} X^{2} S+16 X^{2} \pi^{2}-\frac{56}{9} X^{2}+\frac{40}{3} X Y^{2}\right. \\
& +\frac{88}{3} X Y S+\frac{880}{9} X Y+\frac{220}{3} X S+\frac{88}{3} X \pi^{2}+\frac{1442}{9} X+4 Y^{4}-\frac{88}{3} Y^{2} S \\
& \left.+16 Y^{2} \pi^{2}-\frac{536}{9} Y^{2}+\frac{116}{3} Y \pi^{2}+\frac{484}{9} S^{2}-\frac{44}{3} S \pi^{2}+\frac{5896}{27} S+\frac{58}{9} \pi^{2}+\frac{18028}{81}\right) \frac{t}{u}
\end{aligned}
$$




$$
\begin{aligned}
& +X^{2}\left(X^{2}+4 \pi^{2}\right) \frac{t^{4}}{u^{4}} \\
& +2 X\left(X^{3}+2 X^{2}+4 X \pi^{2}+4 \pi^{2}\right) \frac{t^{3}}{u^{3}} \\
& +\left(7 X^{4}-8 X^{3} Y-\frac{26}{3} X^{3}+12 X^{2} Y^{2}+\frac{88}{3} X^{2} Y-\frac{44}{3} X^{2} S+24 X^{2} \pi^{2}+\frac{28}{9} X^{2}\right. \\
& -8 X Y^{3}-\frac{44}{3} X Y^{2}+\frac{88}{3} X Y S-8 X Y \pi^{2}+\frac{536}{9} X Y+\frac{484}{9} X S-\frac{8}{3} X \pi^{2} \\
& +\frac{2948}{27} X+4 Y^{4}-\frac{88}{3} Y^{2} S+12 Y^{2} \pi^{2}-\frac{536}{9} Y^{2}+\frac{88}{3} Y \pi^{2}+\frac{484}{9} S^{2}-\frac{44}{3} S \pi^{2} \\
& \left.+\frac{5896}{27} S+2 \pi^{4}+\frac{10}{9} \pi^{2}+\frac{18028}{81}\right) \frac{t^{2}}{u^{2}} \\
& +\left(\frac{17}{2} X^{4}-10 X^{3} Y-\frac{7}{3} X^{3}+\frac{15}{2} X^{2} Y^{2}+11 X^{2} Y-\frac{110}{3} X^{2} S+29 X^{2} \pi^{2}\right. \\
& -\frac{122}{3} X^{2}+22 X Y S-5 X Y \pi^{2}+\frac{596}{9} X Y+\frac{814}{9} X S+\frac{107}{3} X \pi^{2} \\
& \left.\left.+\frac{5309}{27} X+\frac{605}{9} S^{2}-11 S \pi^{2}+\frac{7667}{27} S+\frac{5}{4} \pi^{4}+\frac{113}{18} \pi^{2}+\frac{24533}{81}\right)\right\}+\{u \leftrightarrow t\}
\end{aligned}
$$

$$
\begin{aligned}
B= & \left\{6\left(X^{2}-2 X Y+Y^{2}+\pi^{2}\right)\left(X^{2}-2 X Y-2 X+Y^{2}+2 Y+\pi^{2}\right) \frac{t^{4}}{s^{4}}\right. \\
& +\left(72 X^{4}-120 X^{3} Y-356 X^{3}+48 X^{2} Y^{2}+580 X^{2} Y+156 X^{2} \pi^{2}+\frac{1280}{3} X^{2}\right. \\
& +24 X Y^{3}-404 X Y^{2}-144 X Y \pi^{2}-\frac{1184}{3} X Y-392 X \pi^{2}-112 X-24 Y^{4} \\
& \left.+180 Y^{3}-12 Y^{2} \pi^{2}-32 Y^{2}+392 Y \pi^{2}+112 Y+12 \pi^{4}+12 \pi^{2}\right) \frac{t^{2}}{s^{2}} \\
& +\left(-24 X^{4}+144 X^{3} Y+408 X^{3}-48 X^{2} Y^{2}-272 X^{2} Y+120 X^{2} \pi^{2}-64 X^{2}\right. \\
& +96 X Y^{3}+624 X Y^{2}+288 X Y \pi^{2}+\frac{2752}{3} X Y+792 X \pi^{2}+224 X \\
& \left.+144 Y^{2} \pi^{2}+528 Y \pi^{2}+\frac{2200}{3} \pi^{2}\right) \frac{t}{u} \\
& +12 X^{2}\left(X^{2}+4 \pi^{2}\right) \frac{t^{4}}{u^{4}} \\
& +24 X\left(X^{3}+2 X^{2}+4 X \pi^{2}+4 \pi^{2}\right) \frac{t^{3}}{u^{3}} \\
& +\left(84 X^{4}-96 X^{3} Y-104 X^{3}+96 X^{2} Y^{2}+352 X^{2} Y+288 X^{2} \pi^{2}+\frac{1184}{3} X^{2}\right.
\end{aligned}
$$




$$
\begin{aligned}
& \left.-96 X Y \pi^{2}-32 X \pi^{2}+96 Y^{2} \pi^{2}+352 Y \pi^{2}+24 \pi^{4}+\frac{1112}{3} \pi^{2}\right) \frac{t^{2}}{u^{2}} \\
& +\left(42 X^{4}+32 X^{3}+66 X^{2} Y^{2}+416 X^{2} Y+288 X^{2} \pi^{2}+\frac{1808}{3} X^{2}+84 X Y \pi^{2}\right. \\
& \left.\left.+\frac{424}{3} X Y+716 X \pi^{2}+112 X+15 \pi^{4}+666 \pi^{2}+48\right)\right\}+\{u \leftrightarrow t\} \\
& C=\left\{-\left(X^{2}-2 X Y+Y^{2}+\pi^{2}\right)\left(X^{2}-2 X Y-2 X+Y^{2}+2 Y+\pi^{2}\right) \frac{t^{4}}{s^{4}}\right. \\
& +\left(-X^{4}+2 X^{3} Y+\frac{73}{6} X^{3}-3 X^{2} Y^{2}-22 X^{2} Y+\frac{19}{6} X^{2} S-3 X^{2} \pi^{2}\right. \\
& -\frac{185}{18} X^{2}+2 X Y^{3}+22 X Y^{2}+2 X Y \pi^{2}+12 X Y-\frac{83}{9} X S+\frac{32}{3} X \pi^{2} \\
& -\frac{250}{27} X-\frac{19}{2} Y^{3}-\frac{1}{2} Y^{2} S+Y^{2} \pi^{2}-\frac{127}{18} Y^{2}-\frac{31}{3} Y S-\frac{32}{3} Y \pi^{2}-\frac{242}{9} Y \\
& \left.-\frac{88}{9} S^{2}-\frac{976}{27} S-\frac{1}{2} \pi^{4}-2 \pi^{2}-\frac{2752}{81}\right) \frac{t^{2}}{s^{2}} \\
& +\left(-4 X^{4}-4 X^{3} Y-27 X^{3}+6 X^{2} Y^{2}+12 X^{2} Y-X^{2} S-18 X^{2} \pi^{2}\right. \\
& -\frac{127}{9} X^{2}-4 X Y^{3}-11 X Y^{2}+2 X Y S-4 X Y \pi^{2}-\frac{148}{9} X Y-\frac{62}{3} X S \\
& -\frac{149}{3} X \pi^{2}-\frac{484}{9} X+\frac{16}{3} Y^{2} S-2 Y^{2} \pi^{2}+\frac{80}{9} Y^{2}-16 Y \pi^{2}-\frac{176}{9} S^{2} \\
& \left.-S \pi^{2}-\frac{1952}{27} S+\pi^{4}-\frac{247}{9} \pi^{2}-\frac{5504}{81}\right) \frac{t}{u} \\
& -2 X^{2}\left(X^{2}+4 \pi^{2}\right) \frac{t^{4}}{u^{4}} \\
& -4 X\left(X^{3}+2 X^{2}+4 X \pi^{2}+4 \pi^{2}\right) \frac{t^{3}}{u^{3}} \\
& +\left(-7 X^{4}-\frac{28}{3} X^{3}-\frac{16}{3} X^{2} Y+\frac{8}{3} X^{2} S-28 X^{2} \pi^{2}-\frac{52}{3} X^{2}+\frac{8}{3} X Y^{2}\right. \\
& -\frac{16}{3} X Y S-\frac{80}{9} X Y-\frac{176}{9} X S-\frac{64}{3} X \pi^{2}-\frac{976}{27} X+\frac{16}{3} Y^{2} S+\frac{80}{9} Y^{2} \\
& \left.-\frac{16}{3} Y \pi^{2}-\frac{176}{9} S^{2}+\frac{8}{3} S \pi^{2}-\frac{1952}{27} S-\frac{40}{3} \pi^{2}-\frac{5504}{81}\right) \frac{t^{2}}{u^{2}} \\
& +\left(-4 X^{4}+2 X^{3} Y-\frac{13}{2} X^{3}-\frac{3}{2} X^{2} Y^{2}-2 X^{2} Y+\frac{73}{6} X^{2} S-15 X^{2} \pi^{2}\right. \\
& -\frac{131}{18} X^{2}-4 X Y S+X Y \pi^{2}-\frac{97}{9} X Y-\frac{269}{9} X S-\frac{94}{3} X \pi^{2}-\frac{1936}{27} X \\
& \left.\left.-\frac{220}{9} S^{2}+2 S \pi^{2}-\frac{2791}{27} S-\frac{1}{4} \pi^{4}-\frac{367}{18} \pi^{2}-\frac{9337}{81}\right)\right\}+\{u \leftrightarrow t\}
\end{aligned}
$$




$$
\begin{aligned}
& D=\left\{-2\left(X^{2}-2 X Y+Y^{2}+\pi^{2}\right)\left(X^{2}-2 X Y-2 X+Y^{2}+2 Y+\pi^{2}\right) \frac{t^{4}}{s^{4}}\right. \\
& +\left(-4 X^{4}+8 X^{3} Y+75 X^{3}-6 X^{2} Y^{2}-\frac{389}{3} X^{2} Y-8 X^{2} \pi^{2}-\frac{446}{3} X^{2}\right. \\
& +\frac{293}{3} X Y^{2}+4 X Y \pi^{2}+\frac{424}{3} X Y+\frac{232}{3} X \pi^{2}+\frac{116}{3} X+2 Y^{4}-43 Y^{3} \\
& \left.+4 Y^{2} \pi^{2}+\frac{22}{3} Y^{2}-\frac{232}{3} Y \pi^{2}-\frac{116}{3} Y-\pi^{4}-4 \pi^{2}\right) \frac{t^{2}}{s^{2}} \\
& +\left(-4 X^{4}-16 X^{3} Y-102 X^{3}+12 X^{2} Y^{2}+\frac{188}{3} X^{2} Y-32 X^{2} \pi^{2}+\frac{44}{3} X^{2}\right. \\
& -8 X Y^{3}-\frac{380}{3} X Y^{2}-24 X Y \pi^{2}-312 X Y-\frac{578}{3} X \pi^{2}-\frac{232}{3} X-4 Y^{2} \pi^{2} \\
& \left.-\frac{332}{3} Y \pi^{2}+2 \pi^{4}-\frac{820}{3} \pi^{2}\right) \frac{t}{u} \\
& -4 X^{2}\left(X^{2}+4 \pi^{2}\right) \frac{t^{4}}{u^{4}} \\
& -8 X\left(X^{3}+2 X^{2}+4 X \pi^{2}+4 \pi^{2}\right) \frac{t^{3}}{u^{3}} \\
& +\left(-14 X^{4}+8 X^{3}-64 X^{2} Y-56 X^{2} \pi^{2}-\frac{424}{3} X^{2}-16 X \pi^{2}-64 Y \pi^{2}\right. \\
& \left.-\frac{400}{3} \pi^{2}\right) \frac{t^{2}}{u^{2}} \\
& +\left(-4 X^{4}-4 X^{3} Y-\frac{35}{3} X^{3}-85 X^{2} Y-26 X^{2} \pi^{2}-206 X^{2}-2 X Y \pi^{2}\right. \\
& \left.\left.-\frac{148}{3} X Y-\frac{484}{3} X \pi^{2}-\frac{116}{3} X-\frac{1}{2} \pi^{4}-\frac{721}{3} \pi^{2}-16\right)\right\}+\{u \leftrightarrow t\}
\end{aligned}
$$$$
E=\left\{\frac{1}{2}\left(X^{2}-2 X Y+Y^{2}+\pi^{2}\right)\left(X^{2}-2 X Y-2 X+Y^{2}+2 Y+\pi^{2}\right) \frac{t^{4}}{s^{4}}\right.
$$$$
+\left(-\frac{1}{2} X^{4}+2 X^{3} Y-X^{3}-3 X^{2} Y^{2}+2 X^{2} Y-\frac{1}{3} X^{2} S-X^{2} \pi^{2}+\frac{32}{9} X^{2}\right.
$$$$
+2 X Y^{3}-2 X Y^{2}+2 X Y \pi^{2}-6 X Y+\frac{10}{9} X S-\frac{2}{3} X \pi^{2}-\frac{22}{27} X-\frac{1}{2} Y^{4}
$$$$
+Y^{3}+\frac{1}{3} Y^{2} S-Y^{2} \pi^{2}+\frac{10}{3} Y^{2}+\frac{2}{3} Y S+\frac{2}{3} Y \pi^{2}+\frac{34}{9} Y+\frac{8}{9} S^{2}+\frac{80}{27} S
$$$$
\left.-\frac{1}{2} \pi^{4}+\pi^{2}+\frac{236}{81}\right) \frac{t^{2}}{s^{2}}
$$$$
+\left(X^{4}+6 X^{3}-\frac{4}{3} X^{2} Y+\frac{2}{3} X^{2} S+4 X^{2} \pi^{2}+\frac{20}{3} X^{2}+\frac{2}{3} X Y^{2}-\frac{4}{3} X Y S\right.
$$$$
+\frac{4}{3} X S+\frac{34}{3} X \pi^{2}+\frac{68}{9} X+\frac{4}{3} Y \pi^{2}+\frac{16}{9} S^{2}+\frac{2}{3} S \pi^{2}+\frac{160}{27} S
$$ 


$$
\begin{aligned}
& \left.+\frac{22}{3} \pi^{2}+\frac{472}{81}\right) \frac{t}{u} \\
& +X^{2}\left(X^{2}+4 \pi^{2}\right) \frac{t^{4}}{u^{4}} \\
& +2 X\left(X^{3}+2 X^{2}+4 X \pi^{2}+4 \pi^{2}\right) \\
& \frac{t^{3}}{u^{3}}+\left(2 X^{4}+6 X^{3}+8 X^{2} \pi^{2}+\frac{62}{9} X^{2}+\frac{16}{9} X S+12 X \pi^{2}+\frac{80}{27} X+\frac{16}{9} S^{2}\right. \\
& \left.+\frac{160}{27} S+\frac{44}{9} \pi^{2}+\frac{472}{81}\right) \frac{t^{2}}{u^{2}} \\
& +\left(\frac{1}{2} X^{4}-X^{3} Y+\frac{4}{3} X^{3}+\frac{3}{4} X^{2} Y^{2}-X^{2} S+\frac{3}{2} X^{2} \pi^{2}+\frac{28}{9} X^{2}-\frac{1}{2} X Y \pi^{2}\right. \\
& +\frac{5}{9} X Y+\frac{22}{9} X S+\frac{14}{3} X \pi^{2}+\frac{218}{27} X+\frac{20}{9} S^{2}+\frac{254}{27} S+\frac{1}{8} \pi^{4} \\
& \left.\left.+\frac{37}{9} \pi^{2}+\frac{1049}{81}\right)\right\}+\{u \leftrightarrow t\} \\
& F=\left\{-\left(X^{2}-2 X Y+Y^{2}+\pi^{2}\right)\left(X^{2}-2 X Y-2 X+Y^{2}+2 Y+\pi^{2}\right) \frac{t^{4}}{s^{4}}\right. \\
& +\left(X^{4}-4 X^{3} Y-2 X^{3}+6 X^{2} Y^{2}+\frac{14}{3} X^{2} Y+2 X^{2} \pi^{2}+6 X^{2}-4 X Y^{3}\right. \\
& -\frac{14}{3} X Y^{2}-4 X Y \pi^{2}+\frac{4}{3} X Y-\frac{4}{3} X \pi^{2}+\frac{4}{3} X+Y^{4}+2 Y^{3}+2 Y^{2} \pi^{2}-\frac{22}{3} Y^{2} \\
& \left.+\frac{4}{3} Y \pi^{2}-\frac{4}{3} Y+\pi^{4}-2 \pi^{2}\right) \frac{t^{2}}{s^{2}} \\
& +\left(-2 X^{4}-4 X^{3}-\frac{8}{3} X^{2} Y-8 X^{2} \pi^{2}-\frac{44}{3} X^{2}+\frac{8}{3} X Y^{2}+\frac{80}{3} X Y-\frac{28}{3} X \pi^{2}\right. \\
& \left.-\frac{8}{3} X+\frac{8}{3} Y \pi^{2}+16 \pi^{2}\right) \frac{t}{u} \\
& -2 X^{2}\left(X^{2}+4 \pi^{2}\right) \frac{t^{4}}{u^{4}} \\
& -4 X\left(X^{3}+2 X^{2}+4 X \pi^{2}+4 \pi^{2}\right) \frac{t^{3}}{u^{3}} \\
& +\left(-4 X^{4}-12 X^{3}-16 X^{2} \pi^{2}-\frac{4}{3} X^{2}-24 X \pi^{2}+\frac{8}{3} \pi^{2}\right) \frac{t^{2}}{u^{2}} \\
& +\left(-X^{4}+2 X^{3} Y-\frac{4}{3} X^{3}-\frac{3}{2} X^{2} Y^{2}+2 X^{2} Y-3 X^{2} \pi^{2}+\frac{46}{3} X^{2}\right. \\
& \left.\left.+X Y \pi^{2}+2 X Y+\frac{4}{3} X \pi^{2}-\frac{4}{3} X-\frac{1}{4} \pi^{4}+\frac{58}{3} \pi^{2}-8\right)\right\}+\{u \leftrightarrow t\}
\end{aligned}
$$




$$
\begin{aligned}
G= & \left\{3\left(X^{2}-2 X Y+Y^{2}+\pi^{2}\right)\left(X^{2}-2 X Y-2 X+Y^{2}+2 Y+\pi^{2}\right) \frac{t^{4}}{s^{4}}\right. \\
& -3\left(X^{2}-2 X Y+2 X+Y^{2}-2 Y+\pi^{2}-2\right)\left(X^{2}-2 X Y-2 X+Y^{2}+2 Y+\pi^{2}\right) \frac{t^{2}}{s^{2}} \\
& +\left(6 X^{4}+24 X^{3}+24 X^{2} \pi^{2}+36 X^{2}+48 X \pi^{2}+24 X+24 \pi^{2}\right) \frac{t}{u} \\
& +6 X^{2}\left(X^{2}+4 \pi^{2}\right) \frac{t^{4}}{u^{4}} \\
& +12 X\left(X^{3}+2 X^{2}+4 X \pi^{2}+4 \pi^{2}\right) \frac{t^{3}}{u^{3}} \\
& +\left(12 X^{4}+36 X^{3}+48 X^{2} \pi^{2}+36 X^{2}+72 X \pi^{2}+24 \pi^{2}\right) \frac{t^{2}}{u^{2}} \\
& +\left(3 X^{4}-6 X^{3} Y+6 X^{3}+\frac{9}{2} X^{2} Y^{2}+9 X^{2} \pi^{2}+6 X^{2}-3 X Y \pi^{2}+6 X Y\right. \\
& \left.\left.+12 X \pi^{2}+12 X+\frac{3}{4} \pi^{4}+6 \pi^{2}+24\right)\right\}+\{u \leftrightarrow t\}
\end{aligned}
$$

We see that although we expect the finite piece to contain polylogarithms, they are all predicted by the infrared singular structure and are obtained by expanding Eq. (3.2) through to $\mathcal{O}(\epsilon)$. This is because the polylogarithms appear as the $\mathcal{O}(\epsilon)$ and $\mathcal{O}\left(\epsilon^{2}\right)$ terms in the expansion of the box integral in $D=6$ and must be multiplied by an infrared singular term to contribute at $\mathcal{O}(1)$. At $\mathcal{O}(1)$, the interference of one box graph with another only collects the $\mathcal{O}(1)$ terms in each and therefore yields only logarithms.

\section{Summary}

In this paper we presented analytic expressions for the $\mathcal{O}\left(\alpha_{s}^{4}\right)$ QCD corrections to the $2 \rightarrow 2$ gluon-gluon scattering process due to the self-interference of the one-loop amplitude in the $\overline{\mathrm{MS}}$ scheme. Throughout we employed conventional dimensional regularisation.

The renormalised matrix elements are infrared divergent and contain poles down to $\mathcal{O}\left(1 / \epsilon^{4}\right)$. The pole structure of the one-loop contribution is described by Eq. (3.2) using the formalism of Catani [12] while analytic formulae for the finite part according to the colour decomposition of Eq. (3.22) are given in Eqs. (3.23) to (3.29). The interference of the tree-level diagrams with the one-loop graphs are expressed in terms of the one-loop bubble graph in $D=4-2 \epsilon$ dimensions and the one-loop box graph in $D=6-2 \epsilon$ dimensions for which series expansions around $\epsilon=0$ are provided in Appendix A.

The results presented here, together with those previously computed for quarkquark scattering [16, 18, 17], quark-gluon scattering [19] and gluon-gluon scatter- 
ing [20] form a complete set of virtual matrix elements for parton-parton scattering at $\mathcal{O}\left(\alpha_{s}^{4}\right)$. They are vital ingredients for the next-to-next-to-leading order predictions for jet cross sections in hadron-hadron collisions.

The next step is to combine these matrix elements with the tree-level $2 \rightarrow$ $4[30,31,32,33]$, the one-loop $2 \rightarrow 3[34,35,36]$ contributions making sure that the infrared singularities are cancelled. This is a challenging task and a systematic procedure needs to be established. However, recent progress in determining the singular limits of tree-level matrix elements when two particles are unresolved [37, 38] and the soft and collinear limits of one-loop amplitudes [39, 29, 40], together with the analytic cancellation of the infrared singularities in the somewhat simpler case of $e^{+} e^{-} \rightarrow$ photon + jet at next-to-leading order [41], suggest that the technical problems may soon be solved for generic $2 \rightarrow 2$ scattering processes.

Initial state radiation complicates the issue and the colinear singularities from the incoming partons must be factorised into the parton density functions. The evolution of the parton density functions must also be known to an accuracy matching the hard scattering matrix element and requires knowledge of the three-loop splitting functions. Using the existing three-loop order moments [42, 43, 44], Van Neerven and Vogt have provided accurate parameterisations of the splitting functions in $x$ space $[45,46]$ which are now starting to be implemented in the global analyses [47].

Finally, and most importantly for phenomenological applications, an extension of the numerical next-to-leading order two jet programs $[1,2]$ to next-to-next-to-leading order must be developed. We note that Refs. [48, 49] have provided next-to-leading order programs for three jet production that form a natural starting point. We are therefore optimistic that these issues will shortly be resolved thereby enabling next-to-next-to-leading order QCD estimates of jet production in hadron collisions.

\section{Acknowledgements}

M.E.T. acknowledges financial support from CONACyT and the CVCP. We gratefully acknowledge the support of the British Council and German Academic Exchange Service under ARC project 1050. This work was supported in part by the EU Fourth Framework Programme 'Training and Mobility of Researchers', Network 'Quantum Chromodynamics and the Deep Structure of Elementary Particles', contract FMRX-CT98-0194 (DG-12-MIHT).

\section{A. One-loop master integrals}

In this appendix, we list the expansions for the one-loop box integrals in $D=6-2 \epsilon$. We remain in the physical region $s>0, u, t<0$, and write coefficients in terms of logarithms and polylogarithms that are real in this domain. More precisely, we 
use the notation of Eq. (3.21) to define the arguments of the logarithms and the polylogarithms are defined as

$$
\begin{aligned}
& \operatorname{Li}_{n}(w)=\int_{0}^{w} \frac{d t}{t} \operatorname{Li}_{n-1}(t) \quad \text { for } n=2,3,4 \\
& \operatorname{Li}_{2}(w)=-\int_{0}^{w} \frac{d t}{t} \log (1-t) .
\end{aligned}
$$

Using the standard polylogarithm identities [50], we retain the polylogarithms with arguments $x, 1-x$ and $(x-1) / x$, where

$$
x=-\frac{t}{s}, \quad y=-\frac{u}{s}=1-x, \quad z=-\frac{u}{t}=\frac{x-1}{x} .
$$

We find that the box integrals have the expansion

$$
\begin{aligned}
\operatorname{Box}^{6}(u, t)= & \frac{e^{\epsilon \gamma} \Gamma(1+\epsilon) \Gamma(1-\epsilon)^{2}}{2 s \Gamma(1-2 \epsilon)(1-2 \epsilon)}\left(\frac{\mu^{2}}{s}\right)^{\epsilon}\left\{\frac{1}{2}\left[(X-Y)^{2}+\pi^{2}\right]\right. \\
& +2 \epsilon\left[\operatorname{Li}_{3}(x)-X \operatorname{Li}_{2}(x)-\frac{1}{3} X^{3}-\frac{\pi^{2}}{2} X\right] \\
& -2 \epsilon^{2}\left[\operatorname{Li}_{4}(x)+Y \operatorname{Li}_{3}(x)-\frac{1}{2} X^{2} \operatorname{Li}_{2}(x)-\frac{1}{8} X^{4}-\frac{1}{6} X^{3} Y+\frac{1}{4} X^{2} Y^{2}\right. \\
- & \left.\left.\frac{\pi^{2}}{4} X^{2}-\frac{\pi^{2}}{3} X Y-\frac{\pi^{4}}{45}\right]+(u \leftrightarrow t)\right\}+\mathcal{O}\left(\epsilon^{3}\right),
\end{aligned}
$$

and

$$
\begin{aligned}
& \operatorname{Box}^{6}(s, t)= \frac{e^{\epsilon \gamma} \Gamma(1+\epsilon) \Gamma(1-\epsilon)^{2}}{2 u \Gamma(1-2 \epsilon)(1-2 \epsilon)}\left(-\frac{\mu^{2}}{u}\right)^{\epsilon}\left\{\left(X^{2}+2 i \pi X\right)\right. \\
&++\left(-2 \operatorname{Li}_{3}(x)+2 X \operatorname{Li}_{2}(x)-\frac{2}{3} X^{3}+2 Y X^{2}-\pi^{2} X+2 \zeta_{3}\right) \\
&\left.\quad+i \pi\left(2 \operatorname{Li}_{2}(x)+4 Y X-X^{2}-\frac{\pi^{2}}{3}\right)\right] \\
&+\epsilon^{2}\left[\left(2 \operatorname{Li}_{4}(z)+2 \operatorname{Li}_{4}(y)-2 Y \operatorname{Li}_{3}(x)-2 X \operatorname{Li}_{3}(y)+\left(2 X Y-X^{2}-\pi^{2}\right) \operatorname{Li}_{2}(x)\right.\right. \\
&\left.\quad+\frac{1}{3} X^{4}-\frac{5}{3} X^{3} Y+\frac{3}{2} X^{2} Y^{2}+\frac{2}{3} \pi^{2} X^{2}-2 \pi^{2} X Y+2 Y \zeta_{3}+\frac{1}{6} \pi^{4}\right) \\
& \quad+i \pi\left(-2 \operatorname{Li}_{3}(x)-2 \operatorname{Li}_{3}(y)+2 Y \operatorname{Li}_{2}(x)+\frac{1}{3} X^{3}-2 X^{2} Y+3 X Y^{2}\right. \\
&\left.\left.\left.-\frac{\pi^{2}}{3} Y+2 \zeta_{3}\right)\right]\right\}+\mathcal{O}\left(\epsilon^{3}\right) .
\end{aligned}
$$

$\operatorname{Box}^{6}(s, u)$ is obtained from Eq. (A.4) by exchanging $u$ and $t$.

Finally, the one-loop bubble integral in $D=4-2 \epsilon$ dimensions is given by

$$
\operatorname{Bub}(s)=\frac{e^{\epsilon \gamma} \Gamma(1+\epsilon) \Gamma(1-\epsilon)^{2}}{\Gamma(2-2 \epsilon) \epsilon}\left(-\frac{\mu^{2}}{s+i 0}\right)^{\epsilon} .
$$




\section{References}

[1] S.D. Ellis, Z. Kunzst and D.E. Soper, Phys. Rev. Lett. 69 (1992) 3615 [hepph/9208249].

[2] W.T. Giele, E.W.N. Glover and D.A. Kosower, Phys. Rev. D52 (1995) 1486 [hep$\mathrm{ph} / 9412338]$.

[3] V.A. Smirnov, Phys. Lett. B460 (1999) 397 [hep-ph/9905323].

[4] J.B. Tausk, Phys. Lett. B469 (1999) 225 [hep-ph/9909506].

[5] V.A. Smirnov and O.L. Veretin, Nucl. Phys. B566 (2000) 469 [hep-ph/9907385].

[6] C. Anastasiou, T. Gehrmann, C. Oleari, E. Remiddi and J.B. Tausk, Nucl. Phys. B580 (2000) 577 [hep-ph/0003261].

[7] T. Gehrmann and E. Remiddi, Nucl. Phys. Proc. Suppl. 89 (2000) 251 [hep$\mathrm{ph} / 0005232]$.

[8] C. Anastasiou, J.B. Tausk and M.E. Tejeda-Yeomans, Nucl. Phys. Proc. Suppl. 89 (2000) 262 [hep-ph/0005328].

[9] C. Anastasiou, E.W.N. Glover and C. Oleari, Nucl. Phys. B575 (2000) 416, Erratumibid. B585 (2000) 763 [hep-ph/9912251].

[10] R.J. Gonsalves, Phys. Rev. D28 (1983) 1542;

G. Kramer and B. Lampe, J. Math. Phys. 28 (1987) 945.

[11] C. Anastasiou, E.W.N. Glover and C. Oleari, Nucl. Phys. B572 (2000) 307 [hepph/9907494].

[12] S. Catani, Phys. Lett. B427 (1998) 161 [hep-ph/9802439].

[13] Z. Bern, L. Dixon and D.A. Kosower, JHEP 0001 (2000) 027 [hep-ph/0001001].

[14] Z. Bern, L. Dixon and A. Ghinculov, Phys. Rev. D63 (2001) 053007 [hep-ph/0010075].

[15] T.K. Gehrmann and E. Remiddi, Nucl. Phys. B580 (2000) 485 [hep-ph/9912329]; hep-ph/0008287; hep-ph/0101124.

[16] C. Anastasiou, E.W.N. Glover, C. Oleari and M.E. Tejeda-Yeomans, hep-ph/0010212.

[17] C. Anastasiou, E.W.N. Glover, C. Oleari and M.E. Tejeda-Yeomans, hep-ph/0012007.

[18] C. Anastasiou, E.W.N. Glover, C. Oleari and M.E. Tejeda-Yeomans, hep-ph/0011094.

[19] C. Anastasiou, E.W.N. Glover, C. Oleari and M.E. Tejeda-Yeomans, hep-ph/0101304.

[20] E.W.N. Glover, C. Oleari and M.E.Tejeda-Yeomans, hep-ph/0102201.

[21] R.K. Ellis and J.C. Sexton, Nucl. Phys. B269 (1986) 445. 
[22] P. Nogueira, J. Comput. Phys. 105 (1993) 279.

[23] O.V. Tarasov, Phys.Rev.D54 (1996) 6479 [hep-ph/9606018], Nucl.Phys.B502 (1997) 455 [hep-ph/9703319].

[24] K.G. Chetyrkin, A.L. Kataev and F.V. Tkachov, Nucl. Phys. B174 (1980) 345 ;

K.G. Chetyrkin and F.V. Tkachov, Nucl. Phys. B192 (1981) 159.

[25] P. Cvitanovic, P.G. Lauwers and P.N. Scharbach, Nucl. Phys. B186 (1981) 165.

[26] F.A. Berends and W.T. Giele, Nucl. Phys. B294 (1987) 700;

M. Mangano, S.J. Parke and Z. Xu, Nucl. Phys. B298 (1988) 653.

[27] D.A. Kosower, B.-H. Lee and V.P. Nair, Phys. Letts. B201 (1988) 85;

M. Mangano, Nucl. Phys. B309 (1988) 461;

D. Zeppenfeld, Int. J. Mod. Phys. A3 (1988) 2175;

D.A. Kosower, Nucl. Phys. B315 (1989) 391.

[28] Z. Bern and D.A. Kosower, Nucl. Phys. B362 (1991) 389;

Z. Bern, L. Dixon and D.A. Kosower, Ann. Rev. Nucl. Part. Sci. 46 (1996) 109 [hep$\mathrm{ph} / 9602280]$.

[29] Z. Bern, L. Dixon, D.C. Dunbar and D.A. Kosower, Nucl. Phys. B425 (1994) 217 [hep-ph/9403226].

[30] J.F. Gunion and J. Kalinowski, Phys. Rev. D34 (1986) 2119;

S.J. Parke and T.R. Taylor, Nucl. Phys. B269 (1986) 410;

F.A. Berends and W.T. Giele, Nucl. Phys. B294 (1987) 700;

M. Mangano, S.J. Parke and Z. Xu, Nucl. Phys. B298 (1988) 653.

[31] Z. Kunszt, Nucl. Phys. B271 (1986) 333;

S.J. Parke and T.J. Taylor, Phys. Rev. D35 (1987) 313.

[32] J.F. Gunion and Z. Kunszt, Phys. Lett. 159B (1985) 167.

[33] J.F. Gunion and Z. Kunszt, Phys. Lett. 176B (1986) 163.

[34] Z. Bern, L. Dixon, D.A. Kosower, Phys. Rev. Lett 70 (1993) 2677 [hep-ph/9302280].

[35] Z. Bern, L. Dixon and D.A. Kosower, Nucl. Phys. B437 (1995) 259 [hep-ph/9409393].

[36] Z. Kunszt, A. Signer and Z. Trócsányi, Phys. Lett. B336 (1994) 529 [hep-ph/9405386].

[37] J.M. Campbell and E.W.N. Glover, Nucl. Phys. B527 (1998) 264 [hep-ph/9710255];

S. Catani and M. Grazzini, Phys. Lett. 446B (1999) 143 [hep-ph/9810389];

S. Catani and M. Grazzini, Nucl. Phys. B570 (2000) 287 [hep-ph/9908523];

V. Del Duca, A. Frizzo and F. Maltoni, Nucl. Phys. B568 (2000) 211 [hepph/9909464]. 
[38] F.A. Berends and W.T. Giele, Nucl. Phys. B313 (1989) 595;

S. Catani, in Proceedings of the workshop on New Techniques for Calculating Higher Order QCD Corrections, report ETH-TH/93-01, Zurich (1992).

[39] Z. Bern, V. Del Duca and C.R. Schmidt, Phys. Lett. 445B (1998) 168 [hep$\mathrm{ph} / 9810409]$

Z. Bern, V. Del Duca, W.B. Kilgore and C.R. Schmidt, Phys. Rev. D60 (1999) 116001 [hep-ph/9903516];

S. Catani and M. Grazzini, Nucl. Phys. B591 (2000) 435 [hep-ph/0007142].

[40] D.A. Kosower, Nucl. Phys. B552 (1999) 319;

D.A. Kosower and P. Uwer, Nucl. Phys. B563 (1999) 477 [hep-ph/9903515].

[41] A. Gehrmann-De Ridder and E.W.N. Glover, Nucl. Phys. B517 (1998) 269 [hep$\mathrm{ph} / 9707224]$.

[42] S.A. Larin, T. van Ritbergen and J.A.M. Vermaseren, Nucl. Phys. B427 (1994) 41;

S.A. Larin, P. Nogueira, T. van Ritbergen and J.A.M. Vermaseren, Nucl. Phys. B492 (1997) 338 [hep-ph/9605317].

[43] A. Retey and J.A.M. Vermaseren, hep-ph/0007294.

[44] J.A. Gracey, Phys. Lett. B322 (1994) 141 [hep-ph/9401214].

[45] W.L. van Neerven and A. Vogt, Nucl. Phys. B568 (2000) 263 [hep-ph/9907472]; Nucl. Phys. 588 (2000) 345 [hep-ph/0006154].

[46] W.L. van Neerven and A. Vogt, Phys. Lett. B490 (2000) 111 [hep-ph/0007362].

[47] A.D. Martin, R.G. Roberts, W.J. Stirling and R.S. Thorne, Eur. Phys. J. C18 (2000) 117 [hep-ph/0007099].

[48] Z. Trocsanyi, Phys. Rev. Lett. 77 (1996) 2182 [hep-ph/9610499].

[49] W.B. Kilgore and W.T. Giele, Phys. Rev. D55 (1997) 7183 [hep-ph/9610433]; hepph/0009193.

[50] K.S. Kölbig, J.A. Mignaco and E. Remiddi, B.I.T. 10 (1970) 38. 